\title{
A home-based exercise intervention for caregivers of persons with dementia: study protocol for a randomised controlled trial
}

\author{
Wai Chi Chan ${ }^{1 *}$ D, Nicola Lautenschlager ${ }^{2,3,4}$, Briony Dow ${ }^{5}$, Suk Ling Ma ${ }^{6}$, Corine Sau Man Wong ${ }^{1}$ \\ and Linda Chiu Wa Lam ${ }^{6}$
}

\begin{abstract}
Background: Family members, who provide the majority of care for persons with dementia, are especially vulnerable to developing depression. Interventions targeting their depressive symptoms have been proposed but their efficacies vary considerably. It has been suggested that interventions carried out in the home setting and involving both caregivers and care recipients are more efficacious. This study aims to compare the efficacy of a home-based structured exercise programme involving both persons with dementia and their caregivers with nonexercise social contact control in treating depression among caregivers.
\end{abstract}

Methods/design: This is a parallel-group, assessor-blind, randomised controlled trial. A total of 136 caregiver-carerecipient dyads (i.e. 272 participants in total) will be recruited and randomly allocated to either a home-based structured exercise (sitting Tai Chi) group or a social contact control group. The trial comprises a 3-month intervention phase followed by an extended observation phase of another 3 months. All participants will be assessed at baseline, 6th week, 12th week and 24th week. The primary outcome will be the reduction in depression among caregivers as measured by the Hamilton Rating Scale for Depression. The secondary outcomes will be burden, quality of life, cognitive performance and balance ability of the caregivers, as well as the neuropsychiatric symptoms, cognitive function, balance and functional abilities of the persons with dementia. We will also examine whether the brain-derived neurotrophic factor gene modulates mood changes in response to exercise.

Discussion: The findings offer a potential avenue of intervention by providing a low-cost, safe and effective treatment for depression among dementia caregivers, which may in turn also benefit the care recipients.

Trial registration: ClinicalTrials.gov Identifier: NCT02132039, registered on 28 April 2014.

Keywords: Home-based exercise, Tai Chi, Caregivers, Dementia, Depression, Randomised controlled trial

\section{Background}

Dementia affects over 47 million people worldwide [1]. It causes decline in cognitive function, impairment in activities of daily living, and is associated with significant behavioural and psychological symptoms among the persons affected. Since the majority of dementia care is provided by family members [2], it is not surprising that they suffer from a high level of psychological distress

\footnotetext{
* Correspondence: waicchan@hku.hk

'Department of Psychiatry, The University of Hong Kong, Queen Mary

Hospital, Pokfulam, Hong Kong

Full list of author information is available at the end of the article
}

and burden. It is now evident that informal caregivers of persons with dementia are at an elevated risk of developing depression and anxiety, and report poorer quality of life [3-5]. They also experience more stress than those who care for someone with physical disorders [6]. In a local study interviewing family caregivers of older adults with dementia in Hong Kong, they suffered significant stress and reported various negative emotions, conflicts between social roles, and difficulty in providing daily care and managing the care recipients' behaviours [7]. Dementia caregiving has, therefore, emerged as a pressing health care issue. 
In view of this, different interventions have been developed to alleviate depressive symptoms among caregivers of dementia persons. Recent reviews have analysed the efficacies of educational [8] and social support (e.g. befriending and peer support, family support and social network interventions, and support groups) [9], and psychological [10] and Internet-based interventions [11] for caregivers of persons with dementia. Though clinically meaningful, these interventions achieved only small effects [8, 11] or inconsistent results [9]. This may be partly because the programme goals and target groups were not clearly defined. Therefore, it has been suggested that research should focus on subgroups of caregivers such as depressed caregivers [12].

Among interventions for depression, exercise therapy has drawn much attention from researchers and practitioners. Exercise is a subset of physical activity that is planned, structured, repetitive and purposeful, and regular exercise compares favourably to antidepressants as treatment for milder depression [13]. Though its antidepressant mechanism is not yet clear, evidence suggests that exercise improves mood by normalising brainderived neurotrophic factor levels [14], modifying serotonin function and releasing endogenous opioids [15]. More recently, exercise is reported to reduce atrial natriuretic peptide and brain natriuretic peptide, and to enhance copepetin and growth hormone among depressed individuals [16]. In addition, exercise improves one's psychological and social sense of wellbeing [15]. It is, therefore, not surprising that a recent meta-analysis showed that exercise had a large and significant effect on depression (standardised mean deviation adjusted for publication bias $=1.11$ ) with a fail-safe number of 1057 [17].

There has been emerging evidence supporting the effectiveness of exercise in treating depression in dementia caregivers. In a recent study, 17 caregivers participating in regular exercise of moderate intensity experienced less sense of burden and fatigue, and reported better sleep when compared with those in the control group [18]. Encouraging results have also been reported with endurance exercise [19], yoga [20], walking [21], and a combination of various physical activities [22].

However, the number of exercise studies for caregivers remains limited, and the sample sizes involved are usually small. This is probably related to the difficulty for dementia caregivers of participating in centre- or communitybased exercise interventions because they are unable to find someone to take care of the care recipients, and lack time for additional activities [23]. In some previous studies, caregivers also voiced a preference for a programme that both caregiver and care recipient can participate in [22]. Home-based programmes involving both caregivers and care recipients may offer a possible solution [24]. Not only does the home-based dyadic intervention improve treatment adherence [25], it is also more successful in enhancing care-recipient function and reducing caregiver sense of burden [26].

We therefore propose to conduct the following assessor-blind randomised controlled trial of a homebased exercise intervention for both dementia caregivers and their care recipients.

\section{Objectives}

The objectives of the study are as follows:

1. To evaluate the effect of a home-based exercise programme on:

(a). caregivers' depression

(b)caregivers' burden, quality of life, cognitive performance and balance ability

(c).neuropsychiatric symptoms, cognitive function, and balance and functional abilities among persons with dementia

2. To assess whether the brain-derived neurotrophic factor $(B D N F)$ gene modulates mood changes in response to exercise intervention

\section{Methods/design \\ Design}

This is a parallel-group, assessor-blind, randomised controlled trial, which comprises a 3-month intervention phase followed by an extended observation phase lasting for 3 months. Participants will be randomly assigned to either the home-based structured exercise (intervention) or the standard nonexercise social contact (control) group. The study is registered with ClinicalTrials.gov (NCT02132039), and conducted in accordance with the Declaration of Helsinki. Ethics approval has been sought from respective Institutional Review Boards. All results will be reported according to the Consolidated Standards of Reporting Trials (CONSORT) 2010 Statement [27]. A CONSORT flow diagram of the study is depicted in Fig. 1.

\section{Participants}

Caregiver-care-recipient dyads will be recruited from psychogeriatric and geriatric outpatient clinics and various community centres for elders in Hong Kong by convenience sampling. Potential participants will be identified by the research team and screened for eligibility based on the inclusion and exclusion criteria. Informed consent will be obtained from all eligible participants before enrollment.

\section{Inclusion criteria \\ Caregivers:}




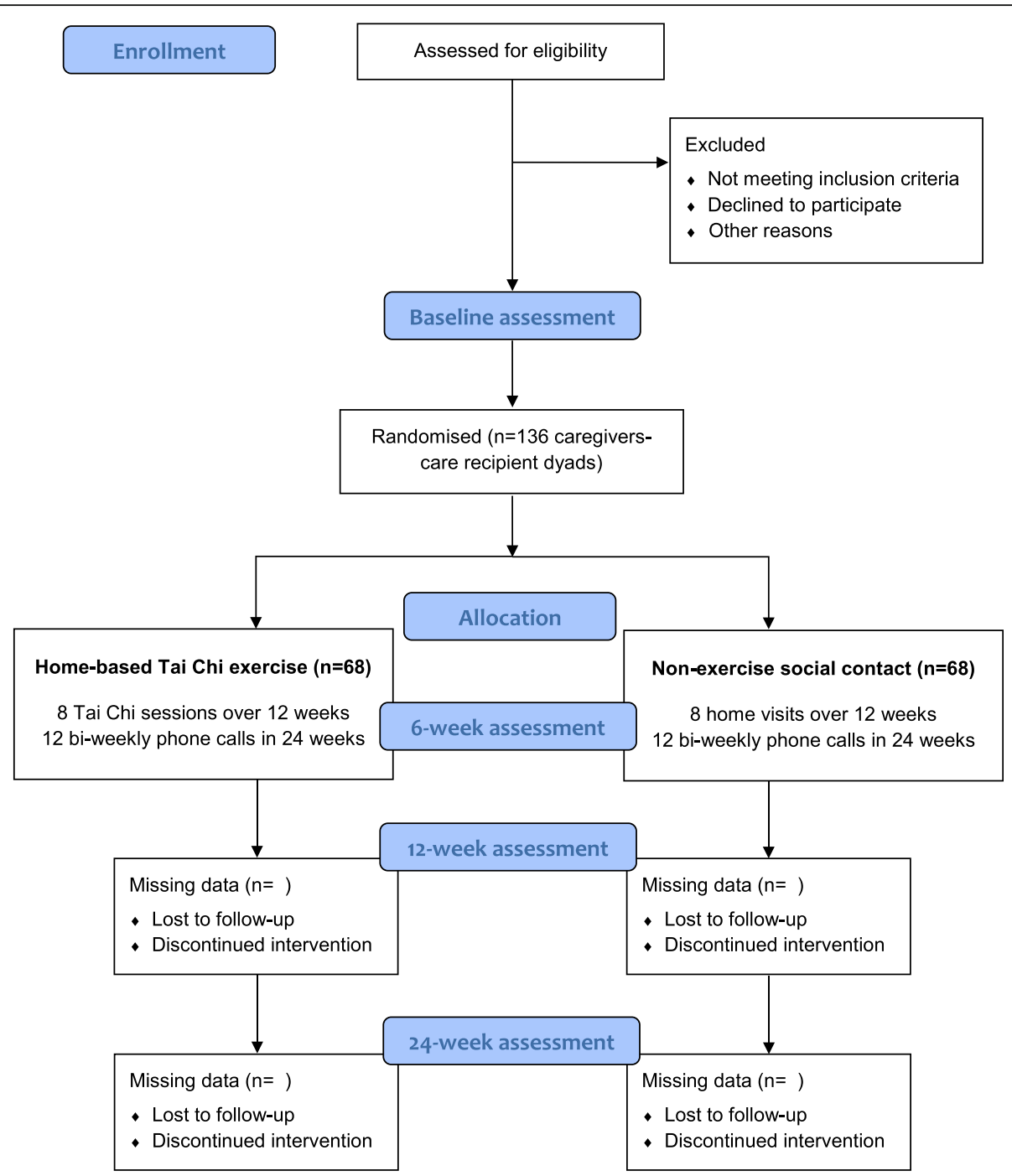

Fig. 1 Consolidated Standards of Reporting Trials (CONSORT) flow diagram of the home-based exercise trial

- Aged 50 years or above

- Informal caregivers, i.e. unpaid family members who offer a substantial amount of care for the persons with dementia

- Geriatric Depression Scale (GDS) [28] score $>0$ but $<8$ (i.e. the local cut-off score for clinically significant depression)

- Understand Chinese

- Receiving antidepressant treatment on a steady dose for at least 3 months (if applicable)

Care recipients:

- Aged 60 years or above

- Dementia diagnosis confirmed by a physician

- Dependence in at least one of the activities of daily living (ADL)

- Mini Mental State Examination (MMSE) [29] score >10

\section{Exclusion criteria}

Both caregivers and care recipients:

- No regular (i.e. at least three times/week) Tai Chi practice or other forms of mind-body exercise such as yoga, qigong, or mindfulness training in the past 6 months

- Presence of any condition which renders participants unsuitable for physical training, for example, severe psychotic symptoms, imminently suicidal, significant orthopaedic problems, or unstable medical conditions

\section{Randomisation and blinding}

Participants will be randomly assigned to either the intervention or the control group using a computergenerated block randomisation sequence (block sizes of 4). Randomisation will be performed by an independent 
team member who will not be involved in the recruitment, assessment, or delivery of the intervention. The allocation sequence will be concealed from the study investigators and research staff in sequentially numbered, opaque, and sealed envelopes. The assessor for clinical parameters will be blinded to the randomisation status, and the trainer who conducts the intervention will be blinded to the assessment results.

\section{Intervention}

The intervention consists of eight home-based sessions of sitting Tai Chi. Tai Chi is a traditional Chinese exercise that combines rhythmic and flowing patterns of bodily movements with coordinated breathing and mindful meditation [30]. It is a moderate-intensity aerobic exercise at about 3 to 6 metabolic equivalents [31]. It has been successfully implemented in the local older population [32] and, as a low impact exercise, it is associated with a lower risk of musculoskeletal injury making it a suitable form of exercise for older adults. Since the current study includes persons with significant cognitive impairment, we adopt sitting Tai Chi which is a simpler version of Tai Chi developed by Hong Kong Polytechnic University [33]. The exercise consists of 12 steps, which include weight shifting in different sitting positions, trunk and upper limb movements, and alternate thigh lift in a smooth and coordinated manner [33].

Four weekly exercise sessions will be arranged over the first 4 weeks, and four more biweekly sessions over the next 8 weeks. Each session will last for $1 \mathrm{~h}$, which starts with $10 \mathrm{~min}$ of warm-up activities followed by $40 \mathrm{~min}$ of sitting Tai Chi practice and, lastly, $10 \mathrm{~min}$ of cool-down exercises. The caregiver-care-recipient dyads will practise Tai Chi in their home under the instruction of a Tai Chi teacher with more than 20 years of teaching experience, and a trained research assistant. In order to monitor the progress and address participants' concerns, biweekly phone contacts will be made during the study period (12 scheduled phone contacts in 24 weeks). On completion of the eight home-based Tai Chi sessions, participants will be given a manual and training video with detailed instructions of the Tai Chi poses to facilitate their continuation of Tai Chi practices.

A standard nonexercise social contact will be provided to the participants in the control group. Since social contact may be beneficial to one's mood and may contribute to any improvement observed in the intervention group, we provide participants in the control group with a level of social contact equivalent to the intervention group. They will be visited by the research assistant eight times over 12 weeks. The visits involve a series of conversations related to neutral topics that are designed according to the principles of a befriending programme [34]. These topics are structured and standardised, and do not involve any topic concerning physical activity. Similar to the intervention group, 12 biweekly phone calls will be made during the study period to offer a comparable level of social contact.

\section{Primary outcome}

Participants will be assessed at baseline, 6th week, 12th week and 24th week for their responses to intervention. The assessment schedules for caregivers and care recipients are described in Table 1 . The primary outcome of the study is the proportion of participants who are classified as responders by the Hamilton Rating Scale for Depression (HAM-D-17) [35]. HAM-D-17 is a widely used and reliable measure of depressive symptoms. The scores range from 0 to 52 , with higher scores indicating greater depression severity. Response to the intervention is defined as a reduction of the HAM-D-17 total score by $\geq 50 \%$ from baseline to endpoint. Assessors will follow the structured interview guide for the Hamilton Depression Rating Scale (SIGH-D) when administering the HAM-D-17 [36].

Assessment for caregivers only:

1. The Zarit Burden Interview (ZBI) [37] is a 22-item self-administrated questionnaire specially designed to measure the stresses experienced by caregivers of persons with dementia. Caregivers will assess the impact of the patient's disabilities on their life

2. The SF-12 Health Survey (SF-12) [38] measures the health-related quality of life and gives two component scores: a physical component summary (PCS) and a mental component summary (MCS)

3. The Executive Interview (EXIT25) [39] is a 25-item screening instrument developed to assess executive cognitive dysfunction

4. The International Personality Item Pool (IPIP) [40] consists of 50 items assessing the 'Big Five' personality traits: openness to experience, conscientiousness, extraversion, agreeableness, and neuroticism

Assessment for care recipients only:

1. The Cornell Scale for Depression in Dementia (CSDD) [41] is a clinician-rated instrument measuring depressive symptoms in persons with cognitive impairment

2. The Neuropsychiatric Inventory (NPI) [42] assesses the frequency and severity of neuropsychiatric symptoms across 12 domains including psychotic features, mood changes, activities disturbances and vegetative symptoms

3. The Disability Assessment for Dementia (DAD) [43] evaluates functional abilities in both basic and 
Table 1 Assessment schedule for caregivers and care recipients

\begin{tabular}{|c|c|c|c|c|}
\hline & $\begin{array}{l}\text { Baseline } \\
\text { assessment }\end{array}$ & $\begin{array}{l}\text { Week } \\
6\end{array}$ & $\begin{array}{l}\text { Week } \\
12\end{array}$ & $\begin{array}{l}\text { Week } \\
24\end{array}$ \\
\hline \multicolumn{5}{|l|}{ Caregiver only } \\
\hline GDS (screening only) & $\sqrt{ }$ & & & \\
\hline HAM-D-17 & $\sqrt{ }$ & $\sqrt{ }$ & $\sqrt{ }$ & $\sqrt{ }$ \\
\hline ZBI & $\sqrt{ }$ & $\sqrt{ }$ & $\sqrt{ }$ & $\sqrt{ }$ \\
\hline SF-12 & $\sqrt{ }$ & & $\sqrt{ }$ & $\sqrt{ }$ \\
\hline EXIT25 & $\sqrt{ }$ & & $\sqrt{ }$ & $\sqrt{ }$ \\
\hline IPIP & $\sqrt{ }$ & & & \\
\hline \multicolumn{5}{|l|}{ Care recipients only } \\
\hline CSDD & $\sqrt{ }$ & $\sqrt{ }$ & $\sqrt{ }$ & $\sqrt{ }$ \\
\hline NPI & $\sqrt{ }$ & & $\sqrt{ }$ & $\sqrt{ }$ \\
\hline DAD & $\sqrt{ }$ & & $\sqrt{ }$ & $\sqrt{ }$ \\
\hline $\mathrm{MBI}$ & $\sqrt{ }$ & & $\sqrt{ }$ & $\sqrt{ }$ \\
\hline \multicolumn{5}{|l|}{$\begin{array}{l}\text { Caregivers and care } \\
\text { recipients }\end{array}$} \\
\hline MMSE & $\sqrt{ }$ & & $\sqrt{ }$ & $\sqrt{ }$ \\
\hline Digit span & $\sqrt{ }$ & & $\sqrt{ }$ & $\sqrt{ }$ \\
\hline Delayed recall & $\sqrt{ }$ & & $\sqrt{ }$ & $\sqrt{ }$ \\
\hline CVFT & $\sqrt{ }$ & & $\sqrt{ }$ & $\sqrt{ }$ \\
\hline BBS & $\sqrt{ }$ & & $\sqrt{ }$ & $\sqrt{ }$ \\
\hline$F R$ & $\sqrt{ }$ & & $\sqrt{ }$ & $\sqrt{ }$ \\
\hline TUG & $\sqrt{ }$ & & $\sqrt{ }$ & $\sqrt{ }$ \\
\hline Logbook & $\sqrt{ }$ & $\sqrt{ }$ & $\sqrt{ }$ & $\sqrt{ }$ \\
\hline Pedometer & $\sqrt{ }$ & $\sqrt{ }$ & $\sqrt{ }$ & $\sqrt{ }$ \\
\hline BDNF genotype & $\sqrt{ }$ & & & \\
\hline $\begin{array}{l}\text { Body weight and } \\
\text { height }\end{array}$ & $\sqrt{ }$ & & $\sqrt{ }$ & $\sqrt{ }$ \\
\hline $\begin{array}{l}\text { Blood pressure and } \\
\text { pulse }\end{array}$ & $\sqrt{ }$ & & $\sqrt{ }$ & $\sqrt{ }$ \\
\hline
\end{tabular}

BBS Berg Balance Scale, BDNF brain-derived neurotrophic factor, CSDD Cornell Scale for Depression in Dementia, CVFT Category Verbal Fluency Test, DAD Disability Assessment for Dementia, EXIT25 Executive Interview, FR Functional Reach, GDS Geriatric Depression Scale, HAM-D-17 Hamilton Rating Scale for Depression, IPIP International Personality Item Pool, MBI Modified Barthel Index, MMSE Mini Mental State Examination, NPI Neuropsychiatric Inventory, TUG Timed Up and Go, ZBI Zarit Burden Interview

instrumental activities of daily living in people with dementia

4. The Modified Barthel Index (MBI) [44] measures individuals' performance on 10 activities of daily living: eating, personal hygiene, bathing, toileting, dressing, bowel control, bladder control, transfers, walking, and stair climbing

Assessment for both caregivers and care recipients:

1. Sociodemographic information includes age, gender, education level and marital status
2. Lifestyle factors such as smoking, drinking, and sleeping habits. Health-related information like chronic health problems and medications will also be recorded. Resting blood pressure and heart rate will be taken. Body height and weight will also be measured to calculate Body Mass Index

3. Global cognitive function will be measured by the MMSE [29], digit span, delayed recall, and the Category Verbal Fluency Test (CVFT) [45]

4. Balance ability and functional mobility will be measured by the Berg Balance Scale (BBS) [46], the Functional Reach (FR) test [47], and the Timed Up and Go (TUG) test [48]

5. Physical activity and programme adherence will be assessed using logbook recordings. Before each assessment, participants will be asked to wear a pedometer for 1 week. Number of walking steps, history of falls, frequency, duration, and type of exercises during the week will be recorded in the logbook

6. Physiological markers - to examine the role of the $B D N F$ Val66Met polymorphism in the antidepressant effect of exercise, buccal swabs will be collected at the baseline assessment

\section{Sample size calculation}

The sample size is calculated by the proportion of caregivers who respond to the intervention as indicated by the HAM-D-17. Taking reference of the previous study [49], we expect a $30 \%$ reduction in the proportion of participants rated as depressed in the control group with social contact only. Assuming that a further $30 \%$ reduction is achieved by sitting Tai Chi, we need 56 caregivercare-recipient dyads (i.e. 112 participants) in each arm to identify a significant effect at a two-tailed alpha of 0.05 and $80 \%$ power. Allowing for $20 \%$ dropout, the number of dyads in each arm will rise to 68 . A total number of participants of 272 will be recruited.

\section{Statistical analyses}

Baseline differences in demographic and clinical characteristics between the intervention and control groups will be evaluated by chi-square and independent sample $t$ tests. All data will be analysed using the intent-to-treat principle. For outcome measures that are not normally distributed (e.g. the HAM-D-17), log transformation will be applied to perform the analyses. Mixed-effects models will be fitted for each outcome measure using baseline, 6th, 12th and 24th week follow-up data. Possible confounding factors such as sex, age, and use of antidepressant medication will be treated as time-dependent covariate in the mixed-effects models. If applicable, subgroup analysis will be carried out to estimate whether there is any 
gender difference (female versus male caregivers) regarding the effect of the intervention in alleviating depressive symptoms. Fixed effects for time, intervention group, and their interactions will be examined and unstructured covariance structure will be employed to account for the within-subject correlation over time. Levels of significance will be set at $p<0.05$. All analyses will be performed using SPSS version 20.0 (SPSS Inc., Chicago, IL, USA).

\section{Discussion}

Since the prevalence of dementia doubles every 5 years after the age of 60 , population ageing inevitably increases the number of persons with dementia. Hong Kong is no exception to this trend. It is estimated that around one in 10 local older persons are now suffering from dementia [50].

Caregiving for persons with dementia is demanding and challenging. Evidence shows that it adversely affects caregivers' physical [51] as well as mental wellbeing [3-5], including a vulnerability to developing depression. In a review of 10 studies involving 790 caregivers of persons with dementia, more than one fifth of them suffered from a depressive disorder [3]. The prevalence of depressive symptoms among caregivers is even higher [14].

This study will investigate the efficacy of a structured exercise programme in alleviating depressive symptoms among caregivers of persons with dementia. We focus on caregivers with depressive symptoms instead of depressive disorders as the former constitute the largest group of depressed caregivers. Besides, because of ethical concerns, we will refer those who suffer from clinically significant depression to appropriate agencies for psychiatric assessment and treatment.

The intervention is tailor-made for family caregivers in three ways. First, the programme is delivered at participants' homes to facilitate their participation. Second, both caregivers and care recipients will be involved in the study. Third, a moderate-intensity aerobic exercise that has been successfully implemented in the local older population (i.e. sitting Tai Chi) is chosen as the intervention.

Sitting Tai Chi is a safe, low-impact and low-cost exercise intervention. If it is shown to improve caregivers' mood, it will offer a potentially effective treatment alternative for depression among caregivers of persons with dementia. In addition, we expect that home-based exercise will also benefit the care recipients.

\section{Trial status}

The study is still recruiting at the time of submission.

\section{Abbreviations}

ADL: Activities of daily living; BBS: Berg Balance Scale; BDNF: brain-derived neurotrophic factor; CONSORT: Consolidated Standards of Reporting Trials; CSDD: Cornell Scale for Depression in Dementia; CVFT: Category Verbal Fluency Test; DAD: Disability Assessment for Dementia; EXIT25: Executive Interview; FR: Functional Reach; GDS: Geriatric Depression Scale; HAM-D17: Hamilton Rating Scale for Depression; IPIP: International Personality Item Pool; MBI: Modified Barthel Index; MCS: Mental component summary; MMSE: Mini Mental State Examination; NPI: Neuropsychiatric Inventory; PCS: Physical component summary; REC: Research Ethics Committee; SF-12: SF-12 Health Survey; SIGH-D: Structured interview guide for the Hamilton Depression Rating Scale; TUG: Timed Up and Go; ZBI: Zarit Burden Interview

\section{Acknowledgements}

We would like to express our sincere thanks to our Tai Chi teacher Mr. Charles Lee for agreeing to take part in the study. We would also like to thank the social centres and clinics for their valuable support in participant recruitment. In addition, we are grateful to Mrs Eleanor Chan and Mrs Miranda Tung for their advice on the assessment methods.

\section{Funding}

The study is supported by the Health and Medical Research Fund of Food and Health Bureau of the Hong Kong Special Administrative Region Government (Ref: 11121441).

\section{Availability of data and materials}

Not applicable.

\section{Authors' contributions}

WCC conceived the idea for the study, obtained funding, and took primary responsibility for study coordination and manuscript preparation. NL, BD, SLM and LCWL obtained funding and contributed to the concept and design of the study, intervention and outcome assessment. CSMW contributed to the coordination of the study, statistical analysis, and manuscript preparation. All authors read and approved the final manuscript.

\section{Competing interests}

The authors declare that they have no competing interests.

\section{Consent for publication \\ Not applicable.}

\section{Ethics approval and consent to participate}

Ethics approval has been sought from the Institutional Review Board of the University of Hong Kong/Hospital Authority Hong Kong West Cluster (UW 13-453), Joint Chinese University of Hong Kong-New Territories East Cluster Clinical Research Ethics Committee (2014.254-T), New Territories West Cluster Clinical and Research Ethics Committee (REC) (NTWC/CREC/ 1302/14), Kowloon West Cluster REC (KW-EX-15-076-86-12TCM), and Hong Kong East Cluster REC (HKEC-2015-045). Participation in the study is completely voluntary. Participants will be able to withdraw from it at any time. The decision to participate or withdraw will not affect their existing treatment or service received. Caregivers who are found to have significant depressive symptoms or other mental health problems will be referred to appropriate health services for follow-up. All personal information collected will be kept strictly confidential and used for research purposes only. Research team members will be responsible for safekeeping the personal data. Only the principal investigator and designated research staff will have access to the interim results and final trial dataset. Informed consent will be obtained from all eligible participants before enrollment.

\section{Author details}

'Department of Psychiatry, The University of Hong Kong, Queen Mary Hospital, Pokfulam, Hong Kong. ${ }^{2}$ Academic Unit for Psychiatry of Old Age, Department of Psychiatry, University of Melbourne, Melbourne, VIC, Australia. ${ }^{3}$ NorthWestern Aged Mental Health, Royal Park Campus, Parkville, VIC,

Australia. ${ }^{4}$ School of Clinical Neurosciences and the Western Australia Centre and Health and Ageing, University of Western Australia, Perth, WA, Australia.

${ }^{5}$ National Ageing Research Institute, The University of Melbourne, Melbourne, 
VIC, Australia. ${ }^{6}$ Department of Psychiatry, The Chinese University of Hong Kong, Tai Po Hospital, Tai Po, Hong Kong.

\section{Received: 7 May 2016 Accepted: 27 August 2016} Published online: 21 September 2016

\section{References}

1. World Health Organization. The epidemiology and impact of dementia: current state and future trends. Geneva; 2015. Available from: http://www. who.int/mental_health/neurology/dementia/dementia_thematicbrief_ epidemiology.pdf. Accessed date 8 Apr 2016.

2. Chan SW. Family caregiving in dementia: the Asian perspective of a global problem. Dement Geriatr Cogn Disord. 2010;30(6):469-78. doi:10.1159/000322086. Epub 20 Jan 2011.

3. Cuijpers P. Depressive disorders in caregivers of dementia patients: a systematic review. Aging Ment Health. 2005;9(4):325-30.

4. Mahoney R, Regan C, Katona C, Livingston G. Anxiety and depression in family caregivers of people with Alzheimer disease: the LASER-AD study. Am J Geriatr Psychiatry. 2005;13(9):795-801.

5. Ho SC, Chan A, Woo J, Chong P, Sham A. Impact of caregiving on health and quality of life: a comparative population-based study of caregivers for elderly persons and noncaregivers. J Gerontol A Biol Sci Med Sci. 2009;64(8):873-9.

6. Ory MG, Hoffman RR, Yee JL, Tennstedt S, Schulz R. Prevalence and impact of caregiving: a detailed comparison between dementia and nondementia caregivers. Gerontologist. 1999;39(2):177-85.

7. Chan WC, Ng C, Mok CCM, Wong FLF, Pang SL, Chiu HFK. Lived experience of caregivers of persons with dementia in Hong Kong: a qualitative study. East Asian Arch Psychiatry. 2010;20(4):163-8.

8. Jensen M, Agbata IN, Canavan M, McCarthy G. Effectiveness of educational interventions for informal caregivers of individuals with dementia residing in the community: systematic review and meta-analysis of randomised controlled trials. Int J Geriatr Psychiatry. 2015;30(2):130-43. Epub 2014/10/30. eng.

9. Dam AE, de Vugt ME, Klinkenberg IP, Verhey FR, van Boxtel MP. A systematic review of social support interventions for caregivers of people with dementia: are they doing what they promise? Maturitas. 2016;85:117-30. Epub 2016/02/10. eng.

10. Elvish R, Lever S-J, Johnstone J, Cawley R, Keady J. Psychological interventions for carers of people with dementia: a systematic review of quantitative and qualitative evidence. Couns Psychother Res. 2013;13(2): $106-25$

11. Boots LM, de Vugt ME, van Knippenberg RJ, Kempen Gl, Verhey FR. A systematic review of Internet-based supportive interventions for caregivers of patients with dementia. Int J Geriatr Psychiatry. 2014;29(4):331-44. Epub 2013/08/22. eng.

12. Smits CHM, de Lange J, Dröes R-M, Meiland F, Vernooij-Dassen M, Pot AM Effects of combined intervention programmes for people with dementia living at home and their caregivers: a systematic review. Int J Geriatr Psychiatry. 2007;22(12):1181-93.

13. Carek PJ, Laibstain SE, Carek SM. Exercise for the treatment of depression and anxiety. Int J Psychiatry Med. 2011;41(1):15-28.

14. De Fazio P, Ciambrone P, Cerminara G, Barbuto E, Bruni A, Gentile P, et al. Depressive symptoms in caregivers of patients with dementia: demographic variables and burden. Clin Interv Aging. 2015;10:1085-90.

15. Stathopoulou G, Powers MB, Berry AC, Smits JAJ, Otto MW. Exercise interventions for mental health: a quantitative and qualitative review. Clin Psychol Sci Pract. 2006;13(2):179-93,

16. Schuch FB, Deslandes AC, Stubbs B, Gosmann NP, Silva CTB, Fleck MPA. Neurobiological effects of exercise on major depressive disorder: a systematic review. Neurosci Biobehav Rev. 2016;2(61):1-11.

17. Schuch FB, Vancampfort D, Richards J, Rosenbaum S, Ward PB, Stubbs B. Exercise as a treatment for depression: a meta-analysis adjusting for publication bias. J Psychiatr Res. 2016;77:42-51.

18. Hirano A, Suzuki Y, Kuzuya M, Onishi J, Ban N, Umegaki H. Influence of regular exercise on subjective sense of burden and physical symptoms in community-dwelling caregivers of dementia patients: a randomized controlled trial. Arch Gerontol Geriatr. 2011;53(2):e158-63.

19. King AC, Baumann K, O'Sullivan P, Wilcox S, Castro C. Effects of moderateintensity exercise on physiological, behavioral, and emotional responses to family caregiving: a randomized controlled trial. J Gerontol A Biol Sci Med Sci. 2002;57(1):M26-36.
20. Waelde LC, Thompson L, Gallagher-Thompson D. A pilot study of a yoga and meditation intervention for dementia caregiver stress. J Clin Psychol. 2004;60(6):677-87.

21. Lowery D, Cerga-Pashoja A, lliffe S, Thuné-Boyle I, Griffin M, Lee J, et al. The effect of exercise on behavioural and psychological symptoms of dementia: the EVIDEM-E randomised controlled clinical trial. Int J Geriatr Psychiatry. 2014;29(8):819-27.

22. Hill K, Smith R, Fearn M, Rydberg M, Oliphant R. Physical and psychological outcomes of a supported physical activity program for older carers. J Aging Phys Act. 2007;15(3):257-71.

23. Etkin CD, Farran CJ, Barnes LL, Shah RC. Recruitment and enrollment of caregivers for a lifestyle physical activity clinical trial. Res Nurs Health. 2012;35(1):70-81. PubMed Central PMCID: PMC3729020.

24. Gitlin LN, Hodgson NA, Choi SSW. Home-based interventions targeting persons with dementia: what is the evidence and where do we go from here? In: Boltz M, Galvin JE, editors. Switzerland: Dementia care: an evidence-based approach: Springer International Publishing; 2016. p. 167-88.

25. Steinberg M, Leoutsakos JMS, Podewils $L$, Lyketsos CG. Evaluation of a home-based exercise program in the treatment of Alzheimer's disease: the Maximizing Independence in Dementia (MIND) study. Int J Geriatr Psychiatry. 2009;24(7):680-5.

26. Thinnes A, Padilla R. Effect of educational and supportive strategies on the ability of caregivers of people with dementia to maintain participation in that role. Am J Occup Ther. 2011;65(5):541-9.

27. Schulz KF, Altman DG, Moher D, Group C. CONSORT 2010 Statement: updated guidelines for reporting parallel group randomised trials. BMC Med. 2010;8:18. PubMed Central PMCID: PMC2860339.

28. Lee HCB, Chiu HFK, Kwok WY, Leung CM, Kwong PK, Chung DWS. Chinese elderly and the GDS short form: a preliminary study. Clin Gerontologist. 1993;14(2):37-42.

29. Chiu HF, Lee H, Chung W, Kwong P. Reliability and validity of the Cantonese version of Mini-mental State Examination: a preliminary study. J Hong Kong Coll Psychiatry. 1994;4 Suppl 2:25-8.

30. Surveillance and Epidemiology Branch, Department of Health, HKSAR. Tai Chi for health. Non-Communicable Disease Watch. 2012;5(4):1-6.

31. Li JX, Hong Y, Chan KM. Tai chi: physiological characteristics and beneficial effects on health. Br J Sports Med. 2001;35(3):148-56. PubMed Central PMCID: PMC1724328.

32. Lam LC, Chau RC, Wong BM, Fung AW, Tam CW, Leung GT, et al. A 1- year randomized controlled trial comparing mind body exercise (Tai Chi) with stretching and toning exercise on cognitive function in older Chinese adults at risk of cognitive decline. J Am Med Dir Assoc. 2012;13(6):568.e15-20.

33. Lee KY, Jones AY, Hui-Chan CW, Tsang WW. Kinematics and energy expenditure of sitting t'ai chi. J Altern Complement Med. 2011;17(8):665-8. Epub 2011/07/08. eng.

34. Bendall S, Killackey E, Jackson $\mathrm{H}$, Gleeson J. Befriending manual. University of Melbourne: OXYGEN Research Centre; 2003.

35. Zheng YP, Zhao JP, Phillips M, Liu JB, Cai MF, Sun SQ, et al. Validity and reliability of the Chinese Hamilton Depression Rating Scale. Br J Psychiatry. 1988;152:660-4.

36. Williams JB. A structured interview guide for the Hamilton Depression Rating Scale. Arch Gen Psychiatry. 1988;45(8):742-7.

37. Chan TSF, Lam LCW, Chiu HFK. Validation of the Chinese version of the Zarit Burden Interview. Hong Kong J Psychiatry. 2005;15(1):9-13.

38. Lam CLK, Tse EYY, Gandek B. Is the standard SF-12 health survey valid and equivalent for a Chinese population? Qual Life Res. 2005;14(2):539-47.

39. Chan SMS, Chiu FKH, Lam CWL. Correlational study of the Chinese version of the Executive Interview (C-EXIT25) to other cognitive measures in a psychogeriatric population in Hong Kong Chinese. Int J Geriatr Psychiatry. 2006;21(6):535-41.

40. Zheng L, Goldberg LR, Zheng Y, Zhao Y, Tang Y, Liu L. Reliability and concurrent validation of the IPIP Big-Five factor markers in China: consistencies in factor structure between Internet-obtained heterosexual and homosexual samples. Pers Individ Dif. 2008:45(7):649-54.

41. Lin JN, Wang JJ. Psychometric evaluation of the Chinese version of the Cornell Scale for Depression in Dementia. J Nurs Res. 2008;16(3):202-10.

42. Leung VP, Lam LC, Chiu HF, Cummings JL, Chen QL. Validation study of the Chinese version of the Neuropsychiatric Inventory (CNPI). Int J Geriatr Psychiatry. 2001;16(8):789-93.

43. Mok CCM, Siu AMH, Chan WC, Yeung KM, Pan PC, Li S-W. Functional disabilities profile of Chinese elderly people with Alzheimer's disease-a 
validation study on the Chinese version of the disability assessment for dementia. Dement Geriatr Cogn Disord. 2005;20(2-3):112-9.

44. Leung SOC, Chan CCH, Shah S. Development of a Chinese version of the modified Barthel Index - validity and reliability. Clin Rehabil. 2007;21(10):912-22.

45. Mok EHL, Lam LCW, Chiu HFK. Category verbal fluency test performance in Chinese elderly with Alzheimer's disease. Dement Geriatr Cogn Disord. 2004;18(2):120-4.

46. Berg KO, Wood-Dauphinee SL, Williams JI, Maki B. Measuring balance in the elderly: validation of an instrument. Can J Public Health. 1992;83 Suppl 2:S7-11.

47. Duncan PW, Weiner DK, Chandler J, Studenski S. Functional reach: a new clinical measure of balance. J Gerontol. 1990:45(6):M192-7.

48. Podsiadlo D, Richardson S. The timed "Up \& Go": a test of basic functional mobility for frail elderly persons. J Am Geriatr Soc. 1991;39(2):142-8.

49. Kerse N, Hayman KJ, Moyes SA, Peri K, Robinson E, Dowell A, et al. Home-based activity program for older people with depressive symptoms: DeLLITE - a randomized controlled trial. Ann Fam Med. 2010;8(3):214-23. PubMed Central PMCID: PMC2866718.

50. Lam LCW, Tam CWC, Lui WWC, Chan WC, Chan SSM, Wong S, et al. Prevalence of very mild and mild dementia in community-dwelling older Chinese people in Hong Kong. Int Psychogeriatr. 2008;20(1):135-48.

51. Patterson TL, Grant I. Interventions for caregiving in dementia: physical outcomes. Curr Opin Psychiatry. 2003;16(6):629-33.

\section{Submit your next manuscript to BioMed Central} and we will help you at every step:

- We accept pre-submission inquiries

- Our selector tool helps you to find the most relevant journal

- We provide round the clock customer support

- Convenient online submission

- Thorough peer review

- Inclusion in PubMed and all major indexing services

- Maximum visibility for your research

Submit your manuscript at www.biomedcentral.com/submit 